\title{
Revisiting and Re-presenting 1980s Micro Computer Art
}

\author{
Sean Clark \\ Interact Digital Arts Ltd. \\ Leicester, UK \\ seanc@interactdigitalarts.uk
}

\author{
Geoff Davis \\ University of the Arts \\ London, UK \\ geoffdavis5@gmail.com
}

\begin{abstract}
The Computer Arts Archive was established in Leicester, UK in early 2020 in order to preserve and develop the CAS50 Collection, a collection of computer artworks brought together in 2018 and 2019 to help celebrate the 50th anniversary of the establishment of the Computer Arts Society. This paper presents work undertaken to expand the archive through the development of focused collections based around areas that we feel are under-reported in the canon of computer and digital art history. The Micro Arts collection explores computer art made using 1980s "micro computers" such as the BBC Micro and Sinclair ZX Spectrum. The starting point of the collection is the Micro Arts project produced in the 1980s by Geoff Davis and colleagues. We present the work undertaken so far in establishing the collection and discuss issues relating to the capture and documentation of pioneering digital artwork such as this.
\end{abstract}

Computer art. Digital art. Micro computers. 8-bit computers.

\section{INTRODUCTION}

The Computer Arts Archive is a not-for-profit company that collects, exhibits and promotes computer arts for the benefit of artists, audiences, curators, educators and researchers. We collaborate with other collections, museums and galleries to explore the impact of digital culture and ensure that computer art is recognised as a significant contemporary art form with a rich and diverse history. In particular, we work closely with the Computer Arts Society, a member-based organisation founded in 1968.

The archive was incorporated in January 2020 as a Community Interest Company and has a base at the LCB Depot in Leicester, UK. As reported at EVA London 2020 (Clark 2020), the project began in 2018 as a collection of artworks brought together to celebrate the formation of the Computer Arts Society 50 years earlier (Clark 2018; Clark 2019).

While the CAS50 Collection remains the central focus of the archive, we are now expanding the collections to include other areas of computer arts history. In particular, we are looking to highlight areas of interest that are not fully integrated in to the mainstream canon of art history. "Micro Arts" is one such area and this paper introduces the work and the materials collected thus far.

\section{COMPUTER GRAPHICS IN THE HOME}

Prior to the 1980s computers with colour graphics capabilities were largely out of reach of the home user. Graphical workstations could be found in Universities and computer graphics companies, but they were extremely expensive and required specialist skills to operate. Home users were typically limited to much less powerful "micro computers" that used character displays and presented their limited graphical outputs on black and white TVs, or on green-screen monitors.

Things changed rapidly in the early 1980s with the release of a host of home computers with colour pixel displays. Despite still being relatively lowpowered, these devices encouraged a generation of people to learn programming and experiment with computer graphics for the first time. For the UK market, the most significant of these early graphical home computers were the BBC Micro from Acorn (released in 1981) and the ZX Spectrum from Sinclair (1982).

As is often the case, amongst the early users of this new medium, were artists. Artists had, of course, been using computers since the 1950s, but the release of the home graphical computer put the technology in to the hands of many artists and creative people who had never been able to access it before. One such group of artists was Micro Arts. 


\section{THE MICRO ARTS GROUP}

The Micro Arts Group was a London-based computer art organisation founded by Geoff Davis in 1984 (Davis 2019). Its members made use of the new generation of low-cost colour graphical computers to create computer artworks. Although they were not alone in seeing the micro computer as a tool for making art, they are fairly unique in that their work was distributed nationally on cassette tape, via a print magazine and - later using the Prestel Teletext service. They were also well-relied in the consumer computer press. Of the four cassette tapes, three (MA1, MA2 and MA4) were created to run on the Sinclair ZX Spectrum $48 \mathrm{~K}$ and one (MA3) for the BBC Micro.

\subsection{MA1 - Geoff Davis: Abstract Originals}

The MA1 ZX Spectrum cassette was released in 1984 and contained a collection of seven colourcontrollable abstract art programs under the name "Abstract Originals". The programs would generate over time and would rebuild from one to the next, with a menu for order, timings and colour control. As can be seen in Figure 1, the colours and style of the computer graphics have a strong imprint of the computer technology of the time. However, despite these limitations, the images are complex and, when running live, engage the viewer.

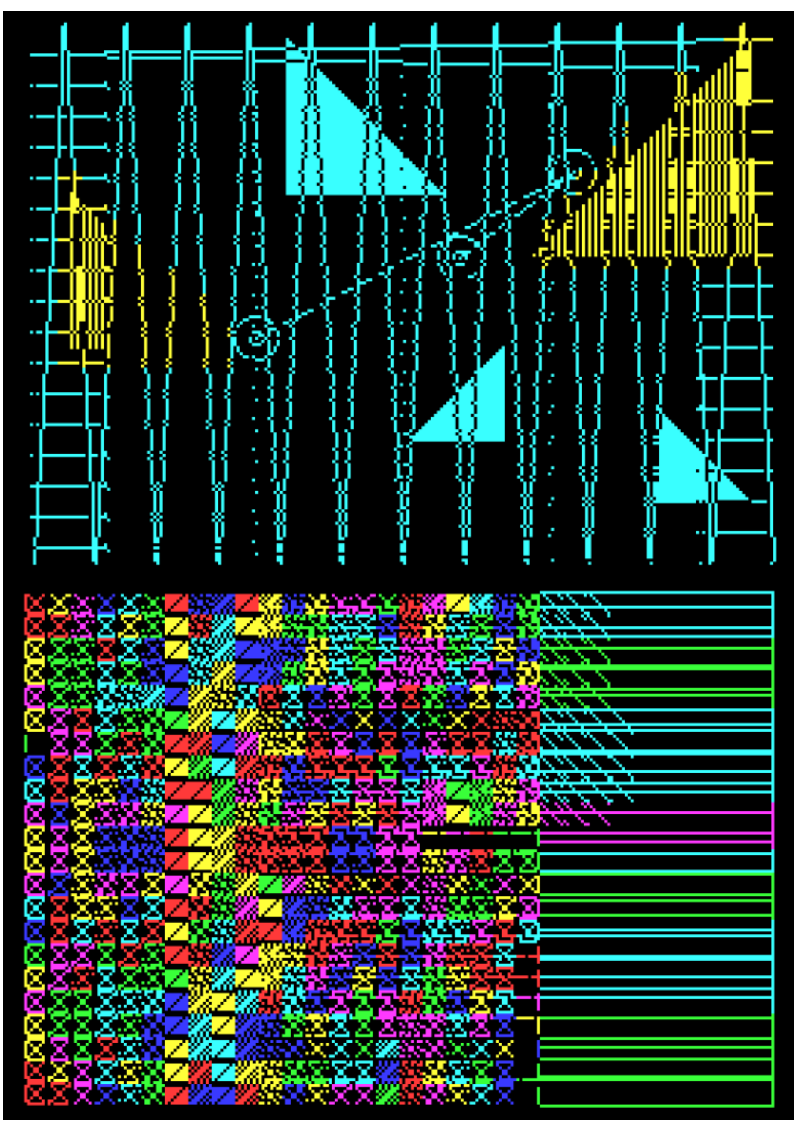

Figure 1. Frames from MA1, by Geoff Davis.

\subsection{MA2 - Geoff Davis: Various Unusual Events}

The MA2 cassette (see Figure 2) was released in 1984 for the ZX Spectrum and contained further art experiments from Geoff Davis. It contained a "slow art" drawing piece that would take almost 2 years to fully complete, other graphical pieces and textbased pieces.

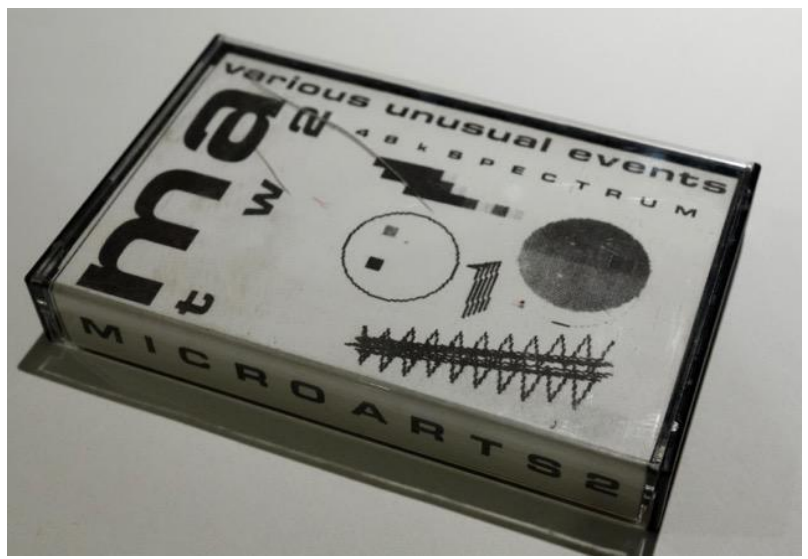

Figure 2. The MA2 cassette for ZX Spectrum 48K.

Again, as can be seen in Figure 3, the colours and fonts are instantly recognisable to people familiar with early 1980 s colour computers.

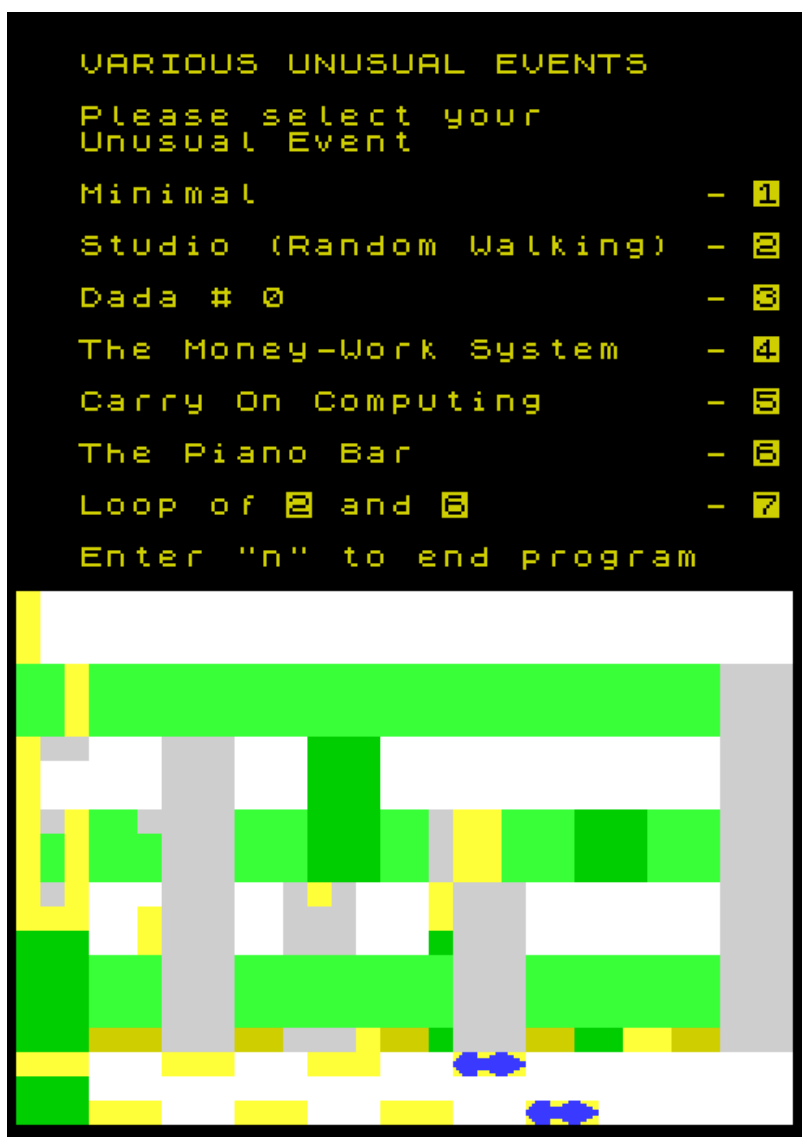

Figure 3. Frames from MA2, by Geoff Davis. 


\subsection{MA3 - Martin Rootes Vol. 1 for the BBC Micro}

Unlike MA1 and MA2, MA3 was made for the BBC Micro and contained artworks by Martin Rootes. Released in 1984 the cassette took a similar form to the previous two, in that multiple artworks could be loaded from the tape as programs and then run on the user's computer.

The BBC Micro was a more powerful device than the ZX Spectrum and the frames shown in Figure 4 have a noticeably different quality, although they still represent typical home computer graphics of the era.

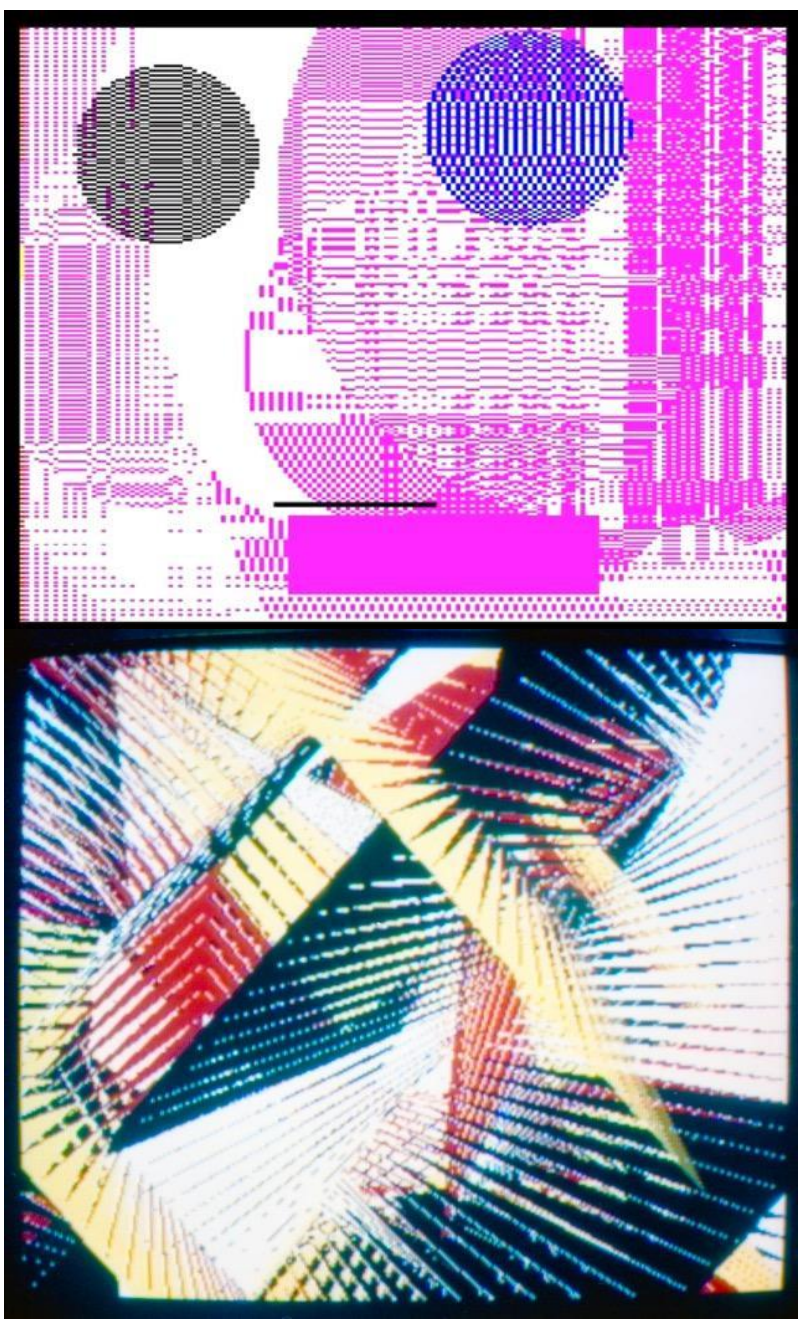

Figure 4. Frames from MA3, by Martin Roots.

\subsection{MA4 - Geoff Davis: Cow Boils Head Story Generator}

MA4 from 1984 marked a return to the $Z X$ Spectrum but, unlike the previous cassettes, took the form of a single, non-graphical, story generator program by Geoff Davis. This is an early example of procedural text generation. The title was a reference to the "Man Bites Dog" definition of a newsworthy story and takes some inspiration from the so-called "Mad Cow Disease" epidemic that was prevalent in the UK at the time. The source text was a short story by Geoff Davis.

The story was generated by applying rules to a set of pre-written snippets of text and was shown onscreen using simple black and white graphics. See Figure 5.

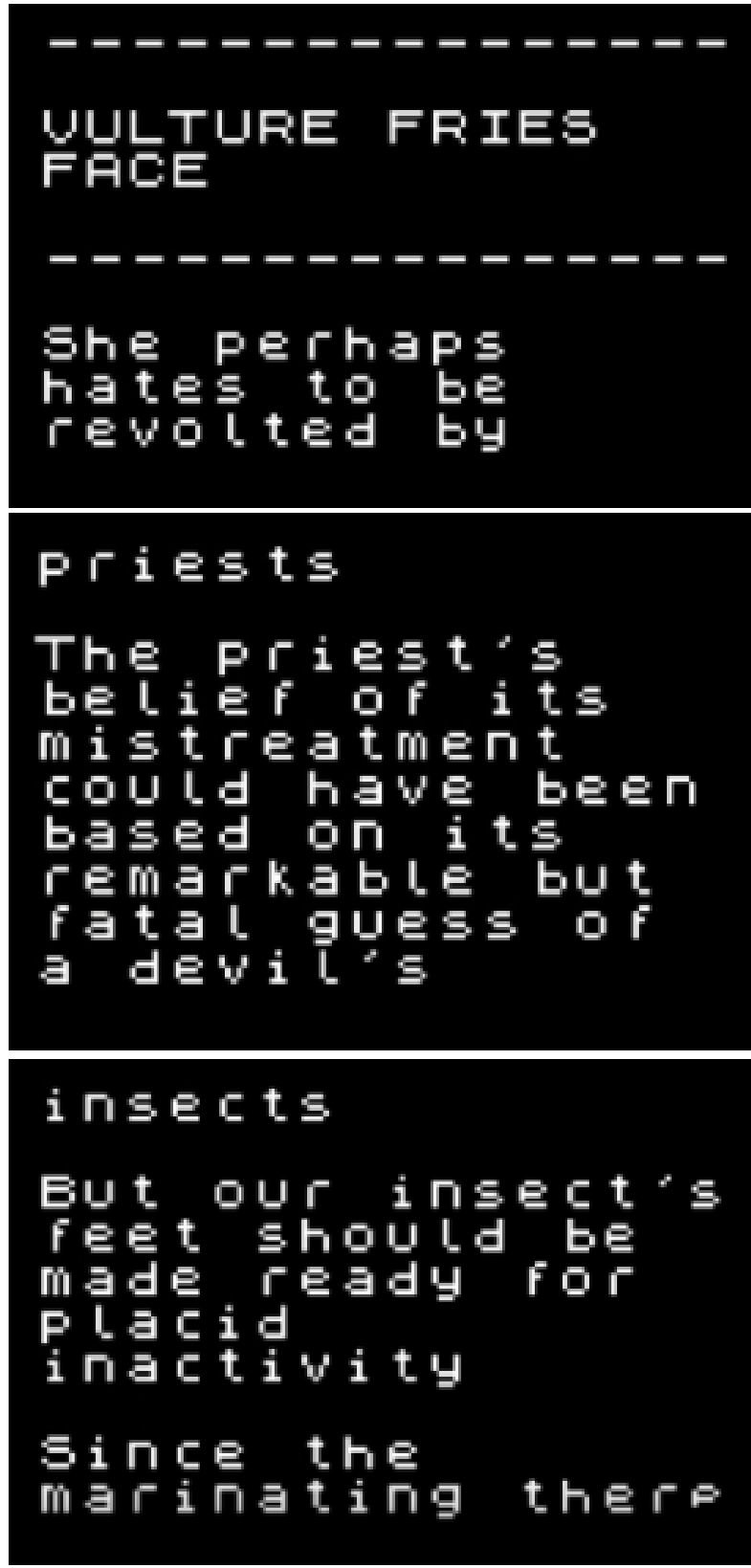

Figure 5. Screen shots from MA4, by Geoff Davis 


\subsection{Micro Arts Magazine - Issue 1}

To accompany the MA1 to MA4 cassettes, a print magazine was published in September 1984 (Davis 1984). Having written for student magazines at the time, it seemed natural for Geoff Davis to look to produce a paper-based publication. A second issue had been prepared but was never published. As such Issue 1 was the sole published output from the Micro Arts Group. It was distributed by Arts Express around galleries and museums.

\subsection{Micro Arts Phase Two - Prestel Teletext}

After four cassettes and a print magazine, the Micro Arts Group was invited to show work on the Prestel Teletext service.

This represented a significant step in terms of the innovative nature of Micro Arts (this was before the World Wide Web had been invented) and it represented an early move into "online art".

However, as Geoff Davis has gone on to note, the lack of subscribers on the service did take some momentum out of the project and there were no more new outputs from the Micro Arts Group after this time.

\section{REVISITING MICRO ARTS}

Given its relatively short history, the Micro Arts Group produced a large amount of artwork. Importantly, Geoff Davis has actively maintained an archive of the project and almost 40 years later much of it can still be run on the original hardware or computer emulators.

This is not the case for much computer software produced on home computers at the time. The fragile nature of cassette tapes and poor quality printing of images has resulted in much work of the time being lost.

Additionally, Geoff Davis has revisited his early work and has created a website devoted to the Micro Arts Group (Davis 2020). In 2019 he also produced a book that documented the work undertaken by the Micro Arts Group (Davis 2019).

It was therefore of great interest when Geoff approached the Computer Arts Archive with the goal of us helping to raise the profile of his collection.
Our original intention was for the Computer Arts Archive to take a copy of all of the surviving materials relating to Micro Arts and arrange an exhibition in mid-2020. This was impacted by the global COVID-19 pandemic and consequently we instead ran an online exhibition in November 2020 as part of the re-scheduled online EVA London conference.

The online exhibition features twelve images taken from the computer artworks in the Micro Arts Collection (Clark 2020) together with information abut the origins of the project. A reconstructed PDF of the Micro Arts Magazine was also included as well as links to videos of the artworks running on a ZX Spectrum emulator. Digital and physical copies of all of these materials are now part of the Computer Arts Archive collections in Leicester.

A launch of the online exhibition took place on 16th November 2020 with speakers including Sean Clark. Nick Lambert, Brian Reffin Smith and Geoff Davis. A recording of this event is available on the Micro Arts Collection web page (ibid).

\section{EXPANDING THE MICRO ARTS COLLECTION}

Having secured a full set of materials from the Micro Arts collection the next stage in our plan is to hold the first physical exhibition of it. This will take place in Leicester in June 2021 with an exhibition of prints from the Micro Arts Collection together with live works running on the Raspberry $\mathrm{Pi} Z \mathrm{ZX}$ Spectrum 48K emulators.

This will be followed by a London exhibition later in 2021, COVID-19 permitting. The London exhibition will be accompanied by a colloquium during which the issues related to collecting digital artwork of this type. We are particularly interested in using this event to engage with other artists who were active in the 1980s and made use of micro computers.

\section{CONCLUSIONS}

Engaging with the Micro Arts Group has given the Computer Arts Archive the opportunity to point a spotlight on an area of computer arts that deserves more attention.

Geoff Davis' extensive archiving of his and his colleagues work from almost 40 years ago is rare and, combined with the volatility of media from the time, we may not be so fortunate in finding complete collections like this in the future. However, the interest in the collection has already garnered suggests that represents a valuable use of our efforts. 


\section{REFERENCES}

Clark, S. (2018) CAS50: Celebrating Fifty Years of the Computer Arts Society. Interact Digital Arts (October 2018) ISBN 978-1-9993103-0-1. Available from: http://bit.ly/2Qwv1tY

Clark, S. (2019) The CAS50 Exhibition and Collection. In: Weinel, J., Bowen, J.P., Diprose, G., and Lambert, N. (eds), EVA London 2019 (Electronic Visualisation and the Arts), 8-11 July 2019. BCS, London.

Clark, S. (2020) The Computer Arts Archive. In: Weinel, J., Bowen, J.P., Diprose, G., and Lambert, N. (eds), EVA London 2020 (Electronic Visualisation and the Arts), 6-9 July 2019. BCS, London.
Clark, S. (2020) The Micro Arts Collection. https://www.computer-artsarchive.com/collections/microarts (retrieved 10 April 2021).

Davis, G. (1984) Micro Arts Magazine Issue 1. Edited and published by Geoff Davis. https://www.computer-artsarchive.com/collections/microarts/magazine (retrieved 10 April 2021).

Davis, G. (2020) The Micro Arts Group. https://microartsgroup.com (retrieved 10 April 2021)

Davis, G. (2019) Micro Arts History 1984-1985. Story Software, London. Available on Amazon. 